\title{
Energy Optimization under Informed Mobility *
}

\author{
Chiping Tang and Philip K. McKinley ${ }^{\dagger}$ \\ Department of Computer Science and Engineering \\ Michigan State University \\ East Lansing, Michigan 48824 \\ \{tangchip,mckinley\}@ cse.msu.edu
}

\begin{abstract}
Energy optimization is important in wireless ad hoc networks, where node battery power is usually limited. Research results show that such a network can exploit controlled node mobility to reduce communication-related energy consumption. However, node movement itself usually consumes energy. In this paper we study the energy optimization problem that accounts for energy costs associated with both communication and physical node movement. We refer to this model as informed mobility. We first review the theoretical foundations on how to reduce total communication energy consumption, as well as increase system lifetime, by combining node movement and transmission power adaptation. Next, we describe and analyze the informed mobility optimization problem. Based on this analysis, we introduce localized algorithms and protocols for informed mobility. We propose iMobif, a flow-based informed mobility framework that collects network information for mobility decision making. We demonstrate how to use iMobif to minimize total communication energy consumption as well as to maximize system lifetime. We compare the performance of iMobif to that of systems with no mobility or only cost-unaware mobility. Simulation results show iMobif is effective in reducing energy consumption relative to such systems.
\end{abstract}

Keywords: mobile ad hoc network, energy optimization, informed mobility, localized algorithm, routing protocol, node position, system lifetime.

\section{Introduction}

In wireless ad hoc networks, wireless nodes communicate with each other by sending data flows either directly or through intermediate relays. The network topology, and specifically the paths of flows, significantly affect communication energy efficiency at individual nodes. Excessive or disproportionate energy consumption among nodes can lead to premature failure of the network. To address this problem, several energy optimization approaches including clustering [1] and topology

* A concise and preliminary version of this paper appeared in the Proceedings of the Second International Workshop on Wireless Ad Hoc Networking (WWAN 2005), Columbus, Ohio, June 2005. This version submitted to TPDS contains more extensive descriptions of the original algorithms plus a new algorithm that optimizes node selection and node movement simultaneously.

$\dagger$ This work was supported in part by U.S. Department of the Navy, Offi ce of Naval Research under Grant No. N00014-01-1-0744. This work was also supported in part by NSF grants CCR-9912407, EIA-0000433, EIA-0130724, and ITR-0313142. 
control [2-4], have been proposed. In these approaches, wireless nodes use techniques such as data aggregation and transmission power adjustment to reduce energy consumption.

If some or all of the nodes in the network are mobile (for example, as in a fleet of microrobots [5]), then it is possible to complement the above energy management strategies with approaches that account for, and even exploit, node mobility. In mobility-aware approaches, the system changes its behavior in response to, or by predicting, node movements. This strategy has been used for topology control [6], energy optimization [7], network throughput improvement [8], and system security improvement [9]. In mobility-aided approaches, the subject of this paper, nodes actually change their locations to optimize certain performance metrics. For example, a mobile sensor node with sufficient energy can move about a sensor field to collect data from other sensor nodes, reducing energy consumption at those nodes. This strategy has been proposed as a means to recover a disconnected topology [10], increase sensor surveillance coverage [11,12] as well as reduce total energy consumption [13-15]. Depending on initial network topology and the power model, such controlled mobility (as opposed to random mobility) can reduce energy consumption by up to $50 \%$ [13].

Despite the fact that node movement itself typically consumes energy, however, many prior studies of controlled mobility have not accounted for this factor. An exception is the work of Goldenberg et al [13], which specifically addresses this tradeoff. Those authors show that it is possible to numerically compare the benefit of mobility with its cost; a node is allowed to move only when the benefit exceeds the cost. Simulation results indicate the benefit outweighs the cost when the number of data bits in the flow surpasses a certain threshold. In this paper we extend that work by designing algorithms and protocols for the collection and distribution of the benefit/cost information, thus enabling local decision-making on controlled mobility.

In this informed mobility optimization problem, the cost and benefit of controlled mobility are weighed in mobility decisions. Factors affecting this tradeoff include the mobility pattern, the network topology, and the data transmission pattern. For such a strategy to be practical, both the cost and benefit calculations should be conducted in a distributed fashion. In addition, such calculations should not include any system parameters that are not measurable by individual nodes. To address this need, we propose iMobif, a flow-based framework to facilitate decision-making related to mobility.

In iMobif, flow sources determine the current mobility strategy and status (enabled or disabled). They inform every node on the flow path of the strategy (and its on-off status) using data packets. 
After receiving a data packet, an intermediate node calculates its new target location according to the current mobility strategy and, if the strategy is enabled, starts moving toward that location. At the same time, the node calculates the cost and benefit of the mobility strategy using local information, aggregates the combined cost-benefit value with the corresponding value in the packet header, and forwards the packet to the next node in the flow path. When the packet eventually arrives at the flow destination, that node can evaluate the overall performance of the mobility strategy based on the aggregate information. If the node determines that a status change is needed, it sends a notification packet back to the source, which updates the mobility strategy status and uses the next data packet to inform all flow nodes.

In addition to changing the mobility strategy status of the existing nodes in a flow, iMobif also supports changing the composition of the flow path. Specifically, iMobif can add new nodes to a flow path so as to further reduce total energy consumption. To achieve this capability, a flow node periodically broadcasts "hello" messages containing the residual energy and location information regarding itself and its flow neighbors. Neighboring nodes that are not currently on the flow path can use this information to determine whether or not they should join the flow path, reporting the cost-benefit comparison results to the flow node. The flow node then chooses the result that maximizes the cost-benefit gain and instructs the chosen node to move towards the flow. The chosen node periodically checks the "hello" messages to determine if the movement remains worthwhile. It stops moving if the expected benefit is not sufficient to counterbalance the cost. Otherwise the node will eventually join the flow.

To our knowledge, this is the first work to address an adaptive mobility strategy based on online cost-benefit information in wireless ad hoc networks. The approach is general in that iMobif can be tuned for different energy optimization goals by changing the mobility strategy and the corresponding cost-benefit aggregate function. In this paper, we demonstrate the effectiveness of iMobif by implementing two different functions, one to minimize total energy consumption and the other to maximize system lifetime (we define system lifetime as the time interval between system initialization and the first time at which a node in the system fails to communicate with others due to energy depletion). While the mobility strategy for the first function is adopted directly from [13], the strategy for the second function is novel. We reported preliminary results on this work in [16].

The remainder of the paper is organized as follows. In Section 2, we review the theoretical foundations on how to reduce total communication energy consumption as well as increase system lifetime by using a combination of node movement and transmission power adaptation. In 
Section 3, we describe the informed mobility optimization problem and propose solutions that use global information. In Section 4 we present the localized algorithms that make informed mobility decisions at individual nodes. In Section 5, we describe the operation of the iMobif framework. We present performance evaluation results in Section 6, and discuss related work in Section 7. In Section 8 , we make concluding remarks and discuss potential research directions.

\section{Preliminaries}

In this section we introduce the energy models used in this study, and describe the positioning of nodes so as to minimize communication energy consumption and maximize system lifetime.

\subsection{Energy Models}

It is well known that the power needed for successful wireless data transmission is determined by the distance between communication entities and the noise level of the communication channel. In this network-layer study, we adopt a transmission power model similar to the one used in [13]. Let $P_{T}(d)$ be the power needed for data transmission across distance $d$, then

$$
P_{T}(d)=a+b d^{\alpha}
$$

where $a, b$, and $\alpha$ are constants dependent on the characteristics of the communication channel. The value of $\alpha$ is usually greater than or equal to 2 . The energy consumption for transmitting $l$ data bits across distance $d$ is

$$
E_{T}(d, l)=l \cdot P_{T}(d)
$$

In addition to transmission energy consumption, we also consider the energy consumption for node mobility, or mobility cost. Of course, mobility cost is dependent on the actual trace of node movement. For simplicity, we adopt a distance proportional cost model similar to the one used in [13]. In this model, the mobility cost $E_{M}(d)$ can be calculated from the distance traversed, $d$ :

$$
E_{M}(d)=k d
$$

where $k$ is a constant dependent on the environment and the mass of the mobile node. The distance proportional model is reasonable for wheeled vehicles (see discussion in [13] for details). 


\subsection{Optimal Node Positions}

In this study we assume that the source and destination nodes of a flow are stationary, but that other nodes are free to move to new locations. We note that in many situations, topology constraints may limit node movement. Such a constraint may be characteristic of the application itself. For example, in a sensor network it might be required that every physical area within the sensing range be covered by at least one sensor node. On the other hand, the constraint might relate to the performance of low-level communication protocols. For example, a limit may be placed on the physical density of nodes so as to reduce channel contention and mutual interference. Evaluating the performance of the proposed methods under such constraints is deferred to a future study. In this paper, the only constraint imposed on the network topology is that it remain connected. Under this assumption, let us begin by presenting two theorems on optimal node configurations.

THEOREM 1 [13]. To minimize total transmission energy consumption, the optimal positions of all $N$ nodes in a one-to-one flow must lie entirely on the straight line between the source node $n_{0}$ and the destination node $n_{N-1}$. Furthermore, all the nodes should be evenly spaced on the line.

To maximize system lifetime, it is necessary to adjust node positions to balance residual energy. A straightforward strategy is to reduce transmission distance for nodes with low residual energy and increase the transmission distance for nodes with high residual energy. We define bottleneck nodes in a network as those with minimum residual energy.

THEOREM 2. To maximize system lifetime, the optimal positions of all $N$ nodes in a one-toone flow must lie entirely on the straight line between the source node $n_{0}$ and the destination node $n_{N-1}$. Let $\mathbf{x}_{\mathbf{i}}$ and $e_{i}$ be the location and residual energy, respectively, of node $n_{i}$ (the $i^{\text {th }}$ node in the flow). Then for any $0 \leq i<N-1,0 \leq j<N-1, \frac{P_{T}\left(d_{i}\right)}{P_{T}\left(d_{j}\right)}=\frac{e_{i}}{e_{j}}$, where $d_{i}=\left|\mathbf{x}_{\mathbf{i}}-\mathbf{x}_{\mathbf{i}+\mathbf{1}}\right|$ is the distance between nodes $n_{i}$ and $n_{i+1}$.

Proof: Assume node $n_{i}$ is not on the line between $n_{i-1}$ and $n_{i+1}$ in an optimal configuration. We show system lifetime can be increased by moving $n_{i}$ to some point on the line. By moving $n_{i}$ from location $\mathbf{x}_{\mathbf{i}}$ to $\mathbf{x}_{\mathbf{i}}^{\prime}$ on the line, as shown in Figure 1, the distance from $n_{i}$ to $n_{i+1}$ can be reduced without increasing the distance between any other node pairs. Let $d_{i}^{\prime}=\left|\mathbf{x}_{\mathbf{i}}^{\prime}-\mathbf{x}_{\mathbf{i}+\mathbf{1}}\right|<d_{i}$ be the distance between $\mathbf{x}_{\mathbf{i}}^{\prime}$ and $\mathbf{x}_{\mathbf{i}+\mathbf{1}}$, and $M$ be the number of bottleneck nodes in the network. We show how the distance reduction $\left(d_{i}-d_{i}^{\prime}\right)$ can be propagated to all $M$ bottleneck nodes as follows:

1. If $n_{i}$ is a bottleneck node, let $r=d_{i}-\frac{d_{i}-d_{i}^{\prime}}{M}$;

2. otherwise let $r=d_{i}$. 
3. Draw a circle around $\mathbf{x}_{\mathbf{i}}^{\prime}$ with radius $r$; node $n_{i+1}$ falls within this circle since $r \geq d_{i}^{\prime}$.

4. If node $n_{i+2}$ also falls within this circle, move $n_{i+1}$ to $\mathbf{x}_{\mathbf{i}+\mathbf{2}}$;

5. otherwise let $\mathbf{x}_{\mathbf{i}+\mathbf{1}}^{\prime}$ be the point where the line $\mathbf{x}_{\mathbf{i}+\mathbf{1}} \mathbf{x}_{\mathbf{i}+\mathbf{2}}$ intersects the circle; move $n_{i+1}$ to $\mathbf{x}_{\mathbf{i}+\mathbf{1}}^{\prime}$;

As shown in Figure 1, the above approach can always reduce the distance between $n_{i+1}$ and $n_{i+2}$ while maintaining the distance between $n_{i}$ and $n_{i+1}$ (if $n_{i}$ is not a bottleneck node) or reducing the distance between $n_{i}$ and $n_{i+1}$ (if $n_{i}$ is a bottleneck node). Similarly, we can change the location of all $n_{j}, j \neq i$, in this way so that the distance reduction $d_{i}-d_{i}^{\prime}$ is propagated and distributed to all bottleneck nodes, and the distance between any bottleneck node and its downstream node is reduced by at least $\frac{d_{i}-d_{i}^{\prime}}{M}$. Consequently, all bottleneck nodes can reduce their energy consumption and the system lifetime can be increased. This is contradictory to the assumption that the network is already in an optimal configuration. Therefore, all nodes in an optimal configuration should be located on the straight line between $n_{0}$ and $n_{N-1}$.

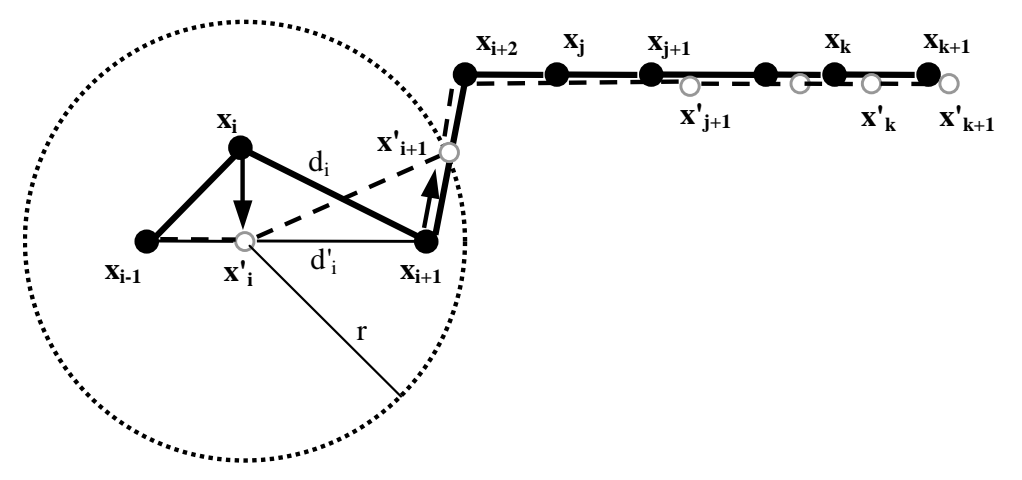

Figure 1. Increasing system lifetime by moving relevant nodes.

Next, we show that for any $0 \leq i<N-1,0 \leq j<N-1, \frac{P_{T}\left(d_{i}\right)}{P_{T}\left(d_{j}\right)}=\frac{e_{i}}{e_{j}}$ when all nodes are located on a straight line. Assume the energy consumption of all activities other than communication is negligible. Let $t_{i}=\frac{e_{i}}{P_{T}\left(d_{i}\right)}$ be the lifetime of node $n_{i}$, and $t$ be the system lifetime. We need to prove that in an optimal configuration $t_{i}=t$ for all $0 \leq i<N$. It is obvious $t_{i} \geq t$. We show $t_{i}=t$ for all $0 \leq i<N$ by contradiction, specifically, that if $t_{j}>t$ for some node $n_{j}$, then the system lifetime can be increased and the current configuration is not optimal.

Let $n_{k}$ be the first bottleneck node for $k>j$ with $t_{k}=t$. Assume there are $M$ such bottleneck nodes. As shown in Figure 1, we can move node $n_{j+1}$ to location $\mathbf{x}_{\mathbf{j}+\mathbf{1}}^{\prime}=\mathbf{x}_{\mathbf{j}+\mathbf{1}}+\delta_{j+1}$ so that the lifetime $t<t_{j}^{\prime}=\frac{e_{j}}{P\left(d_{j}^{\prime}\right)}<t_{j}$. We then shift all nodes from $n_{j+2}$ to $n_{k}$ to the end of $n_{N-1}$ by distance $\delta_{\mathbf{j}+\mathbf{1}}$ so that the distance between them is not changed. Now move node $n_{k+1}$ to location $\mathbf{x}_{\mathbf{k}+\mathbf{1}}^{\prime}=\mathbf{x}_{\mathbf{k}}^{\prime}+d_{k}-\frac{\delta_{j+1}}{M}$ so that $d_{k}^{\prime}=d_{k}-\frac{\delta_{j+1}}{M}$. Then the lifetime of $n_{k}$ is increased to 
$t_{k}^{\prime}=\frac{e_{k}}{P\left(d_{k}^{\prime}\right)}>\frac{e_{k}}{P\left(d_{k}\right)}=t_{k}=t$. Similarly, we can increase the lifetime of all $M$ bottleneck nodes, and thus increase the system lifetime. This is contradictory to the assumption that the current configuration is optimal. Therefore, in an optimal configuration $\frac{e_{i}}{P\left(d_{i}\right)}=t$ for all nodes $n_{i}$, and thus $\frac{P\left(d_{i}\right)}{P\left(d_{j}\right)}=\frac{e_{i}}{e_{j}}$ for any $0 \leq i<N-1,0 \leq j<N-1$.

As shown in the above theorems, energy consumption can be optimized by moving nodes to their optimal positions and setting the transmission power of each node to barely reach the next node in the flow. Obviously, the optimal position of node $n_{i}$ for total energy consumption minimization is,

$$
\mathbf{x}_{\mathbf{i}}=\mathbf{x}_{\mathbf{0}}+\left(\mathbf{x}_{\mathbf{N}-\mathbf{1}}-\mathbf{x}_{\mathbf{0}}\right) \cdot \frac{i}{N-1} \text {. }
$$

Calculating the optimal positions needed to maximize system lifetime is more complicated. From Theorem 2 and the transmission power model, we know, for each $0<i<N$,

$$
\frac{a+b\left(d_{i-1}\right)^{\alpha}}{a+b\left(d_{i}\right)^{\alpha}}=\frac{e_{i-1}}{e_{i}} \text {, and } \sum_{i=0}^{N-2} d_{i}=D=\left|\mathbf{x}_{\mathbf{0}}-\mathbf{x}_{\mathbf{N}-\mathbf{1}}\right| .
$$

The closed-form solutions for $d_{i-1}$ and $d_{i}$ are very complicated or even unattainable for $\alpha>2$. To make the calculations practical, we can use a simple approximation:

$$
\frac{\left(d_{i-1}\right)^{\alpha}}{\left(d_{i}\right)^{\alpha}}=\frac{e_{i-1}}{e_{i}}
$$

Under this simplification, the optimal position of $n_{i}$ for system lifetime maximization is

$$
\mathbf{x}_{\mathbf{i}}=\mathbf{x}_{\mathbf{0}}+\left(\mathbf{x}_{\mathbf{N}-\mathbf{1}}-\mathbf{x}_{\mathbf{0}}\right) \cdot \frac{\sum_{j=0}^{i-1}\left(e_{j}\right)^{\frac{1}{\alpha}}}{\sum_{j=0}^{N-2}\left(e_{j}\right)^{\frac{1}{\alpha}}} \text {, for } 0<i<N-1 .
$$

Next we show how to take both transmission energy consumption and mobility cost into consideration to achieve overall energy optimization.

\section{The Informed Mobility Optimization Problem}

In this section we introduce the informed mobility optimization problem. First we present the definition of the problem. Then we show how to solve the problem for flows with fixed composition. Lastly we discuss the solution for flows with no composition constraint.

\subsection{Problem Definition}

In the informed mobility optimization problem, the position and initial residual energy of each node is given. The goal is to find the optimal node positions, under network topology constraints, that maximize the energy utilization $G(\mathbf{E}, \mathbf{X})$, which is defined as a function on the current node energy set $\mathbf{E}$ and position set $\mathbf{X}$. 


\section{DEFINITION 1. The informed mobility optimization problem.}

Let $\mathbf{N}_{\mathbf{A}}$ be the node set of a mobile ad hoc network $A$. Given the initial energy set $\mathbf{E}$ and position set $\mathbf{X}$, which contains the initial node energy pair $<n_{i}, e_{i}>$ and node position pair $<n_{i}, \mathbf{x}_{\mathbf{i}}>$ for each node $n_{i} \in \mathbf{N}_{\mathbf{A}}$, find the optimal position set $\mathbf{X}^{\prime}\left(<n_{i}, \mathbf{x}_{\mathbf{i}}^{\prime}>\right.$ for each node $\left.n_{i}\right)$ such that the network topology constraint $C\left(\mathbf{X}^{\prime}\right)$ is satisfied and the energy utilization function $G\left(\mathbf{E}^{\prime}, \mathbf{X}^{\prime}\right)$ is maximized. According to the cost model (Section 2.1), this goal is equivalent to maximizing

$$
G\left(\mathbf{E}-E_{M}\left(d\left(\mathbf{X}, \mathbf{X}^{\prime}\right)\right), \mathbf{X}^{\prime}\right)=G\left(\left\{e_{i}-k\left|\mathbf{x}_{\mathbf{i}}-\mathbf{x}_{\mathbf{i}}^{\prime}\right|\right\},\left\{\mathbf{x}_{\mathbf{i}}^{\prime}\right\}\right),
$$

while satisfying $C\left(\left\{\mathbf{x}_{\mathbf{i}}^{\prime}\right\}\right)$, where $\left|\mathbf{x}_{\mathbf{i}}-\mathbf{x}_{\mathbf{i}}^{\prime}\right|$ is the distance between positions $\mathbf{x}_{\mathbf{i}}$ and $\mathbf{x}_{\mathbf{i}}^{\prime}$.

The solution to the informed mobility optimization problem depends on the energy utilization function $G$ and the topology constraint $C$. In this work we study two flow-based sub-problems under the topology constraint (as mentioned above) of preventing network disconnection. Specifically, we assume there exist multiple flows $\mathbf{F}_{\mathbf{i}}$ in network $A$, each of which transmits $\lambda_{i}$ data bits from a source node to a destination node. Let node $n_{(i, j)}$ be the $j^{\text {th }}$ node in flow $\mathbf{F}_{\mathbf{i}} \in \mathbf{F}_{\mathbf{A}}$, where $\mathbf{F}_{\mathbf{A}}$ is the flow set of network $A$. A network node $A$ may be involved in multiple flows. It expends a certain amount of energy to transmit the data bits of each flow (except for the flow in which the node is the destination) to the next node in the flow. According to the transmission power model described in Section 2.1, the minimum transmission energy consumption for each node $n_{i}$ is

$$
E_{T}^{i}(\mathbf{X})=\sum_{j, n_{i}=n_{\left(j, k_{j}\right)} \in \mathbf{F}_{\mathbf{j}}} E_{T}\left(\left|\mathbf{x}_{\left(\mathbf{j}, \mathbf{k}_{\mathbf{j}}\right)}-\mathbf{x}_{\left(\mathbf{j}, \mathbf{k}_{\mathbf{j}}+\mathbf{1}\right)}\right|, \lambda_{j}\right),
$$

and the minimum unit transmission energy consumption for each node $n_{i}$ is

$$
E_{t}^{i}(\mathbf{X})=\sum_{j, n_{i}=n_{\left(j, k_{j}\right)} \in \mathbf{F}_{\mathbf{j}}} E_{T}\left(\left|\mathbf{x}_{\left(\mathbf{j}, \mathbf{k}_{\mathbf{j}}\right)}-\mathbf{x}_{\left(\mathbf{j}, \mathbf{k}_{\mathbf{j}}+\mathbf{1}\right)}\right|, 1\right) .
$$

The first sub-problem seeks to minimize total energy consumption. We define the energy utilization function $G_{1}$ as

$$
G_{1}(\mathbf{E}, \mathbf{X})=\sum_{<n_{i}, e_{i}>\in \mathbf{E}}\left(e_{i}-E_{T}^{i}(\mathbf{X})\right)
$$

in which $e_{i}$ is introduced to reflect the energy consumption of node movement.

The second sub-problem seeks to maximize system lifetime, defined as the number of flow bits transmitted before a node in the flow fails to communicate with others due to energy depletion. We define the energy utilization function $G_{2}$ as

$$
G_{2}(\mathbf{E}, \mathbf{X})=\min _{<n_{i}, e_{i}>\in \mathbf{E}} \frac{e_{i}}{E_{t}^{i}(\mathbf{X})}
$$


The formulae above show the evaluation of the energy utilization functions depends on the flow compositions. Next we introduce the methods for calculating optimal node positions.

\subsection{Solutions for Flows with Fixed Composition}

Section 2.2 showed that the optimal positions of nodes in a flow must lie entirely on the straight line between the flow source and the destination. This observation, however, does not account for the mobility cost. Indeed, it is possible for a node to spend so much energy to move to its "optimal" position, that the energy utilization functions produce lower values than if the node had not moved.

We use an example to illustrate our solutions. In Figure 2, the nodes $\left(e . g ., n_{i}\right)$ move from their initial positions $\left(e . g ., \mathbf{x}_{\mathbf{i}}\right)$ to their "optimal" positions $\left(e . g ., \mathbf{x}_{\mathbf{i}}^{\prime}\right)$ to minimize total energy consumption. At the initial positions the mobility cost is zero, but the transmission energy consumption is high. As the nodes start moving to their "optimal" positions according to the theorems in Section 2.2, the mobility cost increases while the transmission energy consumption decreases. As shown in the figure, at intermediate positions $\left(\right.$ e.g., $\left.\mathbf{x}_{\mathbf{i}}^{\prime \prime}\right)$, the decrease of total transmission distance $\sum_{i}\left|\mathbf{x}_{\mathbf{i}}-\mathbf{x}_{\mathbf{i}+\mathbf{1}}\right|-\sum_{i}\left|\mathbf{x}_{\mathbf{i}}^{\prime \prime}-\mathbf{x}_{\mathbf{i}+\mathbf{1}}^{\prime \prime}\right|$ is monotonic in the distance traveled $\sum_{i}\left|\mathbf{x}_{\mathbf{i}}-\mathbf{x}_{\mathbf{i}}^{\prime \prime}\right|$. The reduction of transmission energy consumption, however, depends on node positions. We propose a simple algorithm shown in Figure 3: if the mobility cost $E_{M}\left(d\left(\mathbf{X}^{\prime}, \mathbf{X}\right)\right)$ is not covered by the energy savings $\sum_{i}\left(E_{T}^{i}(\mathbf{X})-E_{T}^{i}\left(\mathbf{X}^{\prime}\right)\right)$ at the "optimal" positions $\mathbf{X}^{\prime}$, then the original positions $\mathbf{X}$ are the true optimal positions; otherwise, positions $\mathbf{X}^{\prime}$ are the true optimal positions. A similar algorithm can be used to maximize system lifetime.

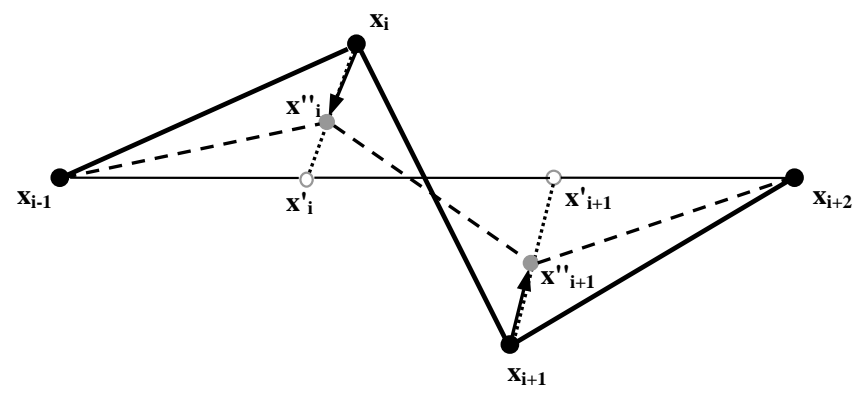

Figure 2. Nodes moving to their optimal positions.

\subsection{Solutions for Flows with Dynamic Composition}

By changing the composition of a flow (e.g., adding new nodes to a flow), the value of the energy utilization functions can be further improved. Again we use an example of minimizing total energy 


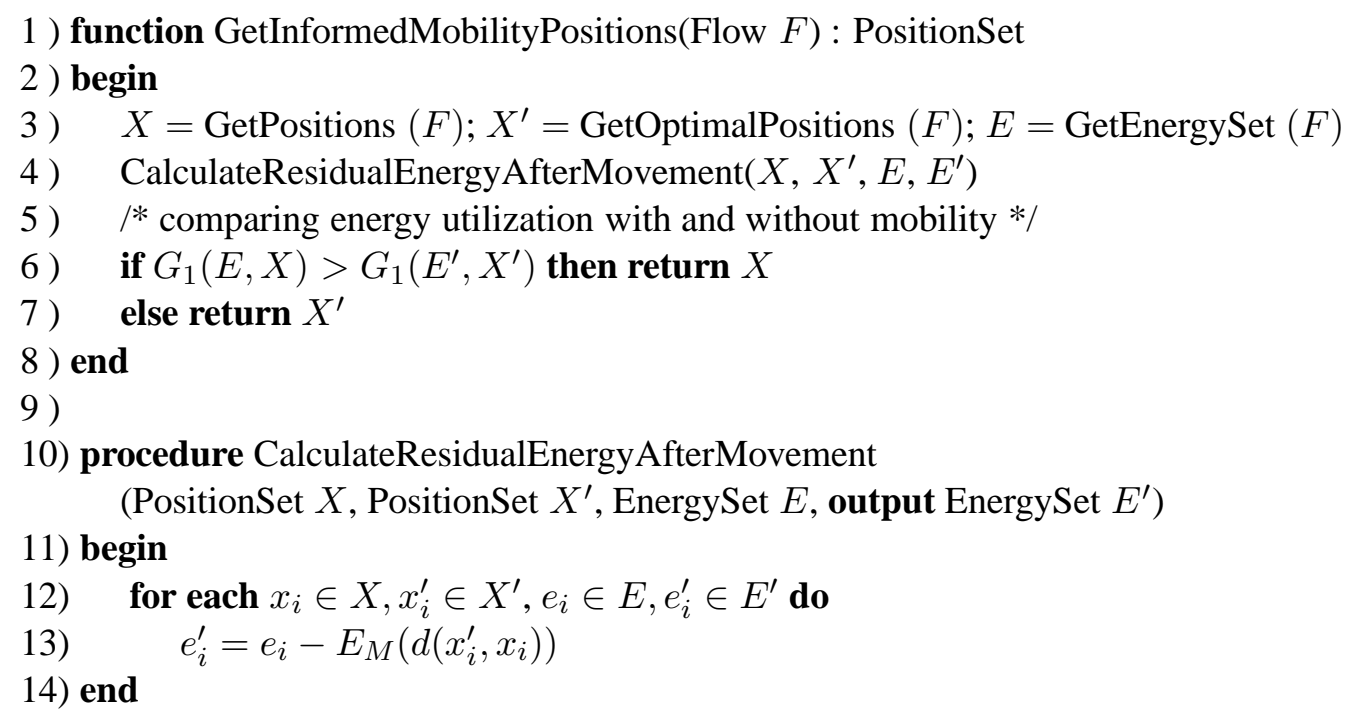

Figure 3. Calculating optimal node positions for fixed composition flows.

consumption to illustrate the solution. Assume flow $\mathbf{F}$ includes $m$ nodes, and the distance between the source and the destination is $D$. Inserting node $n^{\prime}$ into the flow between nodes $n_{i}$ and $n_{i+1}$, and moving nodes to their "optimal" positions according to Theorem 1, the total transmission energy consumption becomes $m \cdot E_{T}\left(\frac{D}{m}, \lambda\right)$. Considering the transmission energy consumption before the insertion, the energy savings is $(m-1) \cdot E_{T}\left(\frac{D}{m-1}, \lambda\right)-m \cdot E_{T}\left(\frac{D}{m}, \lambda\right)$. If the mobility cost is lower than the energy savings, then the total energy consumption is reduced. The algorithm is shown in Figure 4. This approach can also be used to maximize system lifetime by moving the nodes to their "optimal" positions according to Theorem 2 after the insertion.

\subsection{Multiple Flow Considerations}

So far we have discussed the optimal positions for nodes in a single flow. In real scenarios, multiple flows may co-exist in one network, and a given node may be included in multiple flows. Moreover, its optimal position with respect to one flow is likely to be different than with respect to another. A coordination mechanism is needed to combine the results for multiple flows.

We propose a solution based on the results from [13]. We imagine there exists a virtual force for each flow that pushes a node from its current position to its "optimal" position determined by that flow. The strength of the force is proportional to the number of data bits in the flow, and dependent on the distance between the node and its neighboring nodes in the flow. The forces may neutralize 
1 ) procedure JoinFlowUnderInformedMobility(Flow $F$, Node $n$ )

2 ) begin

3) $X=$ GetPositions $(F) ; X^{\prime}=\operatorname{GetOptimalPositions}(F) ; E=\operatorname{GetEnergySet}(F)$

4 ) CalculateResidualEnergyAfterMovement $\left(X, X^{\prime}, E, E^{\prime}\right)$

5 ) $\quad / *$ attempting to insert $n$ into the flow */

6 ) $\quad F_{0}=F ; n$.join $\left(F_{0}\right)$

7 ) $\quad X_{0}=$ GetPositions $\left(F_{0}\right) ; X_{0}^{\prime}=$ GetOptimalPositions $\left(F_{0}\right) ; E_{0}=\operatorname{GetEnergySet}\left(F_{0}\right)$

8 ) $\quad / *$ calculating residual energy assuming $n$ has joined the flow $* /$

9) CalculateResidualEnergyAfterMovement $\left(X_{0}, X_{0}^{\prime}, E_{0}, E_{0}^{\prime}\right)$

10) $\quad / *$ insert $n$ into the flow if the energy utilization increases after the insertion */

11) if $G_{1}\left(E_{0}^{\prime}, X_{0}^{\prime}\right)>G_{1}\left(E^{\prime}, X^{\prime}\right)$ and $G_{1}\left(E_{0}^{\prime}, X_{0}^{\prime}\right)>G_{1}(E, X)$ then $n$.join $(F)$

12) end

Figure 4. Node insertion algorithm.

each other and generate a new force, which pushes the node to its final position. The final position of node $n_{i}$ can be calculated as

$$
\mathbf{x}_{\mathbf{i}}^{\prime}=\frac{\left.\left.\sum_{j, n_{i}=n_{\left(j, k_{j}\right)} \in F_{j}}\left(\left|\mathbf{x}_{\left(\mathbf{j}, \mathbf{k}_{\mathbf{j}}\right)}^{\prime}-\mathbf{x}_{\left(\mathbf{j}, \mathbf{k}_{\mathbf{j}}+\mathbf{1}\right)}\right|^{\alpha-2}+\mid \mathbf{x}_{\left(\mathbf{j}, \mathbf{k}_{\mathbf{j}}\right)}^{\prime}\right)_{\left(\mathbf{j}, \mathbf{k}_{\mathbf{j}}-\mathbf{1}\right)}\right|^{\alpha-2}\right) \lambda_{j} \mathbf{x}_{\left(\mathbf{j}, \mathbf{k}_{\mathbf{j}}\right)}^{\prime}}{\sum_{j, n_{i}=n_{\left(j, k_{j}\right)} \in F_{j}}\left(\left|\mathbf{x}_{\left(\mathbf{j}, \mathbf{k}_{\mathbf{j}}\right)}^{\prime} \mathbf{x}_{\left(\mathbf{j}, \mathbf{k}_{\mathbf{j}}+\mathbf{1}\right)}\right|^{\alpha-2}+\left|\mathbf{x}_{\left(\mathbf{j}, \mathbf{k}_{\mathbf{j}}\right)}^{\prime}-\mathbf{x}_{\left(\mathbf{j}, \mathbf{k}_{\mathbf{j}}-\mathbf{1}\right)}\right|^{\alpha-2}\right) \lambda_{j}},
$$

where $\mathbf{x}_{\left(\mathbf{j}, \mathbf{k}_{\mathbf{j}}\right)}^{\prime}$ is the optimal position of node $n_{i}$ calculated by flow $\mathbf{F}_{\mathbf{j}}$.

After the final target position is obtained, the mobility cost is compared with the aggregate mobility benefit, and node mobility is enabled only when the latter is greater. The algorithm is given in Figure 5.

\section{Localized Algorithms}

The algorithms presented in Section 3 use global information, such as the positions of the source and destination nodes, to make mobility decisions. In real scenarios, it is unlikely that such information is always available at each node. In this section, we propose localized versions of the algorithms. We replace the position and residual energy of all flow nodes with the position and residual energy of the current node and its neighboring nodes in the flow.

\subsection{Solutions for Flows with Fixed Composition}

First, we propose a localized algorithm for flows with fixed composition based on the algorithm in [13]. The basic idea is to have each node constantly adjust its position according to the positions of its neighbors in the flow. In Figure 8, for example, a node chooses as its next target position the 


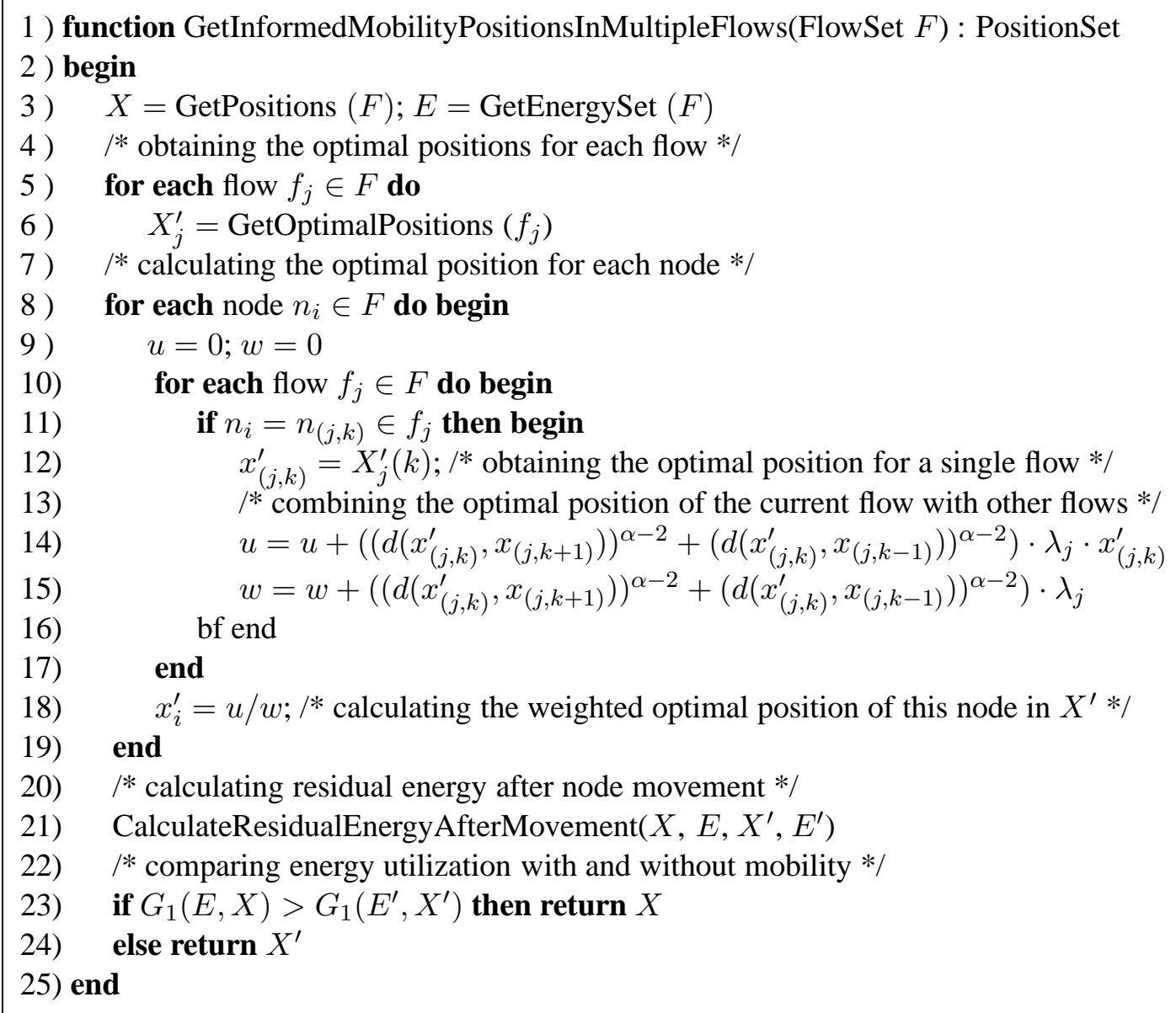

Figure 5. Calculating optimal node positions in multiple flows.

center of the straight line between its two neighbors. It is shown in [13] that the final positions of the nodes will eventually converge to their optimal positions on the straight line. We extend that algorithm by taking mobility cost into consideration: a node periodically calculates the mobility benefit (transmission energy consumption reduction or node lifetime increase) and the mobility cost, based on its current position and the next target position. The algorithm is shown in Figure 6.

To make mobility decisions regarding system-wide metrics such as the total energy consumption or system lifetime, it is necessary to aggregate the mobility benefit and mobility cost at individual nodes. To do so, the algorithm exploits the characteristics of flow-based communication: messages sent from the source to the destination pass through all intermediate nodes. During message propagation, the mobility benefit and cost at each node can be aggregated and placed in a message header. The destination node thus obtains the aggregated mobility benefit and cost of the flow. If the benefit is greater than the cost, then the nodes should move to their next target positions; oth- 
erwise they should maintain their current positions. The destination records the mobility status (to move or not to move) of the flow. In the case of a status change, it sends a notification message back to the source. The source places the current mobility status in message headers so that the flow nodes can obtain the status and enforce mobility accordingly.

This algorithm is "generic" in the sense that it can be applied to various informed mobility problems. Next we will show how to use the generic algorithm to minimize total energy consumption or maximize system lifetime. We note the proposed algorithm is an approximation of the global algorithm described in Section 3. However, the simulation results in Section 6 show the energy performance improvement is significant even though the localized approximation does not always produce an optimal solution.

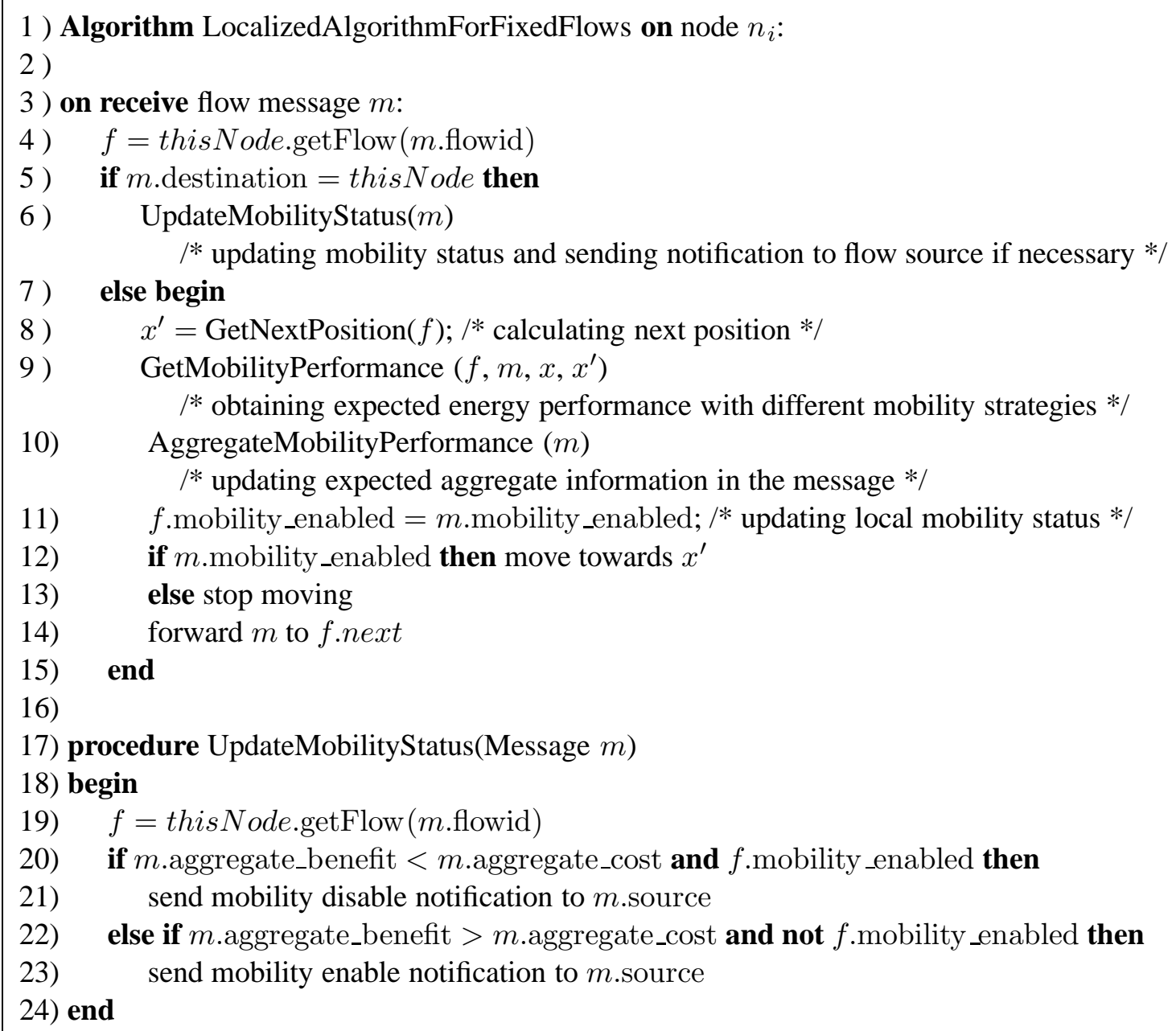

Figure 6. The localized algorithm for fixed flows. 


\subsubsection{Minimizing Total Energy Consumption}

To minimize total energy consumption, it is necessary to calculate next node positions, estimate the expected transmission energy consumption at the current and next positions, estimate the expected energy consumption for moving to next positions, and obtain the total flow energy consumption at the destination. Specifically, each node should implement the functions GetNextPosition(), GetMobilityPerformance(), and AggregateMobilityPerformance() in the generic algorithm. The function implementations are shown in Figure 7.

1 ) function GetNextPosition (Flow $f$ ) : Position

2 ) begin

3 ) return $($ f.prev.x + f.next.x $) / 2$

4 ) end

5 )

6 ) procedure GetMobilityPerformance (Flow $f$, inout Message $m$, Position $x$, Position $x^{\prime}$ )

7 ) begin

8 ) $\quad$ m.benefit $=E_{T}\left(\mid x-\right.$ f.next.x $\left.\mid, \lambda_{f}\right)-E_{T}\left(\mid x^{\prime}-\right.$ f.next.x $\left.\mid, \lambda_{f}\right)$

9) $\left.\quad m \cdot \operatorname{cost}=E_{M}\left(\left|x-x^{\prime}\right|\right)\right)$

10) end

11)

12) procedure AggregateMobilityPerformance (inout Message $m$ )

13) begin

14) $m$.aggregate_benefit $+=m$.benefit

15) $m$.aggregate_cost $+=m \cdot \operatorname{cost}$

16) end

Figure 7. Minimizing total energy consumption using the generic algorithm for fixed flows.

As shown in Figure 8, a node chooses as its next position the center of the straight line between the previous and next nodes in the flow path. As described in Section 5, this information is locally available. The total energy consumption of the flow can be collected using summation operation. We set the benefit to be the difference between transmission energy consumption at the current and next positions, and the cost to be the energy consumption for moving to next positions.

\subsubsection{Maximizing System Lifetime}

To maximize system lifetime, it is necessary to calculate next node positions, estimate expected node lifetime at the current and next positions, and obtain the minimum in-flow node lifetime at the destination. The next node position can be calculated locally using an approximation as described 


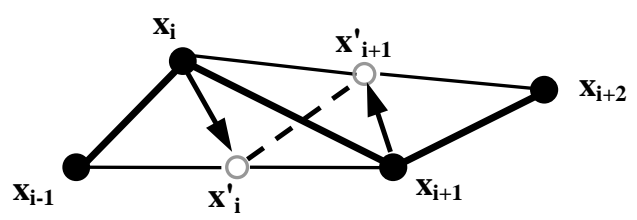

Figure 8. Calculating the new node location that minimizes total energy consumption.

in Section 2.2. The approximation is based on Theorem 2 as well as the transmission power model. The basic idea is to move each node $n_{i}$ from $\mathbf{x}_{\mathbf{i}}$ to a new location $\mathbf{x}_{\mathbf{i}}^{\prime}$ on the line between $\mathbf{x}_{\mathbf{i}-\mathbf{1}}$ and $\mathbf{x}_{\mathbf{i}+\mathbf{1}}$. The distance $d_{i-1}^{\prime}=\left|\mathbf{x}_{\mathbf{i}-\mathbf{1}}-\mathbf{x}_{\mathbf{i}}^{\prime}\right|$ as well as the distance $d_{i}^{\prime}=\left|\mathbf{x}_{\mathbf{i}}^{\prime}-\mathbf{x}_{\mathbf{i}+\mathbf{1}}\right|$ is calculated using the location and residual energy information of the previous and the current node. As described in Section 5, all such information is locally available. The calculation is based on the following equations:

$$
\begin{aligned}
& d_{i-1}^{\prime}+d_{i}^{\prime}=D=\left|\mathbf{x}_{\mathbf{i}-\mathbf{1}}-\mathbf{x}_{\mathbf{i}+\mathbf{1}}\right|, \\
& \frac{a+b\left(d_{i-1}^{\prime}\right)^{\alpha}}{a+b\left(d_{i}^{\prime}\right)^{\alpha}}=\frac{e_{i-1}}{e_{i}} .
\end{aligned}
$$

The first equation ensures that the new location is on the line between the previous and the next nodes, and the second equation is derived from Theorem 2 directly. As described in Section 2.2, closed-form solutions for $d_{i-1}^{\prime}$ and $d_{i}^{\prime}$ are very complicated or even unattainable for $\alpha>2$. Hence, we use a simple approximation:

$$
\begin{aligned}
& \frac{\left(d_{i-1}^{\prime}\right)^{\alpha}}{\left(d_{i}^{\prime}\right)^{\alpha}}=\frac{e_{i-1}}{e_{i}}, \text { so } \\
& \mathbf{x}_{\mathbf{i}}^{\prime}=\mathbf{x}_{\mathbf{i}-\mathbf{1}}+\left(\mathbf{x}_{\mathbf{i}+\mathbf{1}}-\mathbf{X}_{\mathbf{i}-\mathbf{1}}\right) \cdot \frac{\left(e_{i-1}\right)^{\frac{1}{\alpha}}}{\left(e_{i-1}\right)^{\frac{1}{\alpha}}+\left(e_{i}\right)^{\frac{1}{\alpha}}} .
\end{aligned}
$$

The accuracy of this approximation depends on the relative weight between $a$ and $b\left(d_{i}^{\prime}\right)^{\alpha}$. In Section 6 we show $b\left(d_{i}^{\prime}\right)^{\alpha}$ may be much larger than $a$ in real environments, where this approximation is effective in increasing system lifetime.

The minimum lifetime among nodes on the flow path can be collected using the above aggregation procedure, replacing the summation operation with the minimum operation. To make the mobility benefit and cost comparable, we set the benefit to be the system lifetime with mobility, and set the cost to be the system lifetime without mobility. The corresponding function implementations are shown in Figure 9. 


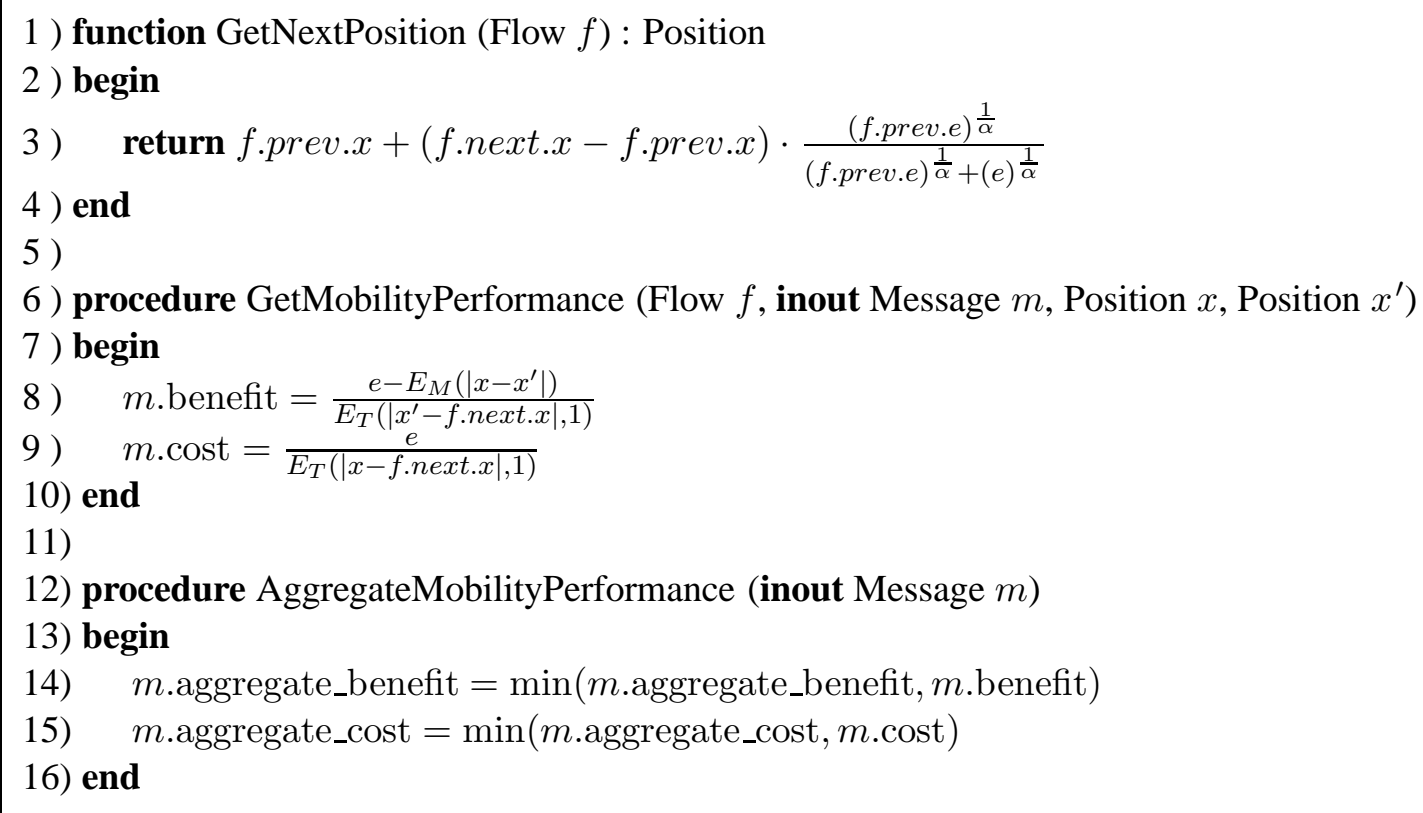

Figure 9. Maximizing system lifetime using the generic algorithm for fixed flows.

\subsection{Solutions for Flows with Dynamic Composition}

Next we describe the localization of the algorithms that change the composition of the flow by inserting new nodes. As described in Section 5, a flow node $n_{i}$ periodically broadcasts the position and residual energy information of itself as well as its neighbors $\left(n_{i-1}, n_{i+1}\right)$ in the flow. A nearby non-flow node $n_{j}$ overhearing this message calculates the mobility benefit and the cost of joining this flow, with the expected position of $n_{j}$ calculated using the function GetNextPosition() introduced in Figure 7 or 9. If the expected gain is greater than the cost, then $n_{j}$ sends the benefit and cost value to $n_{i}$, which may have received similar information from other non-flow nodes. Node $n_{i}$ selects the node $n_{k}$ that maximizes the mobility gain, and tentatively attaches that node to the flow. Node $n_{k}$ periodically checks the mobility benefit and cost of joining the flow. If the mobility benefit is greater than the cost, $n_{k}$ continues to move toward its expected position; otherwise it stops. When $n_{k}$ becomes close enough to $n_{i-1}$ and $n_{i}$ so that it is beneficial for $n_{k}$ to join the flow, $n_{k}$ formally inserts itself into the flow between $n_{i-1}$ and $n_{i}$. A generic algorithm is shown in Figure 10. To use the algorithm, an application implements the function GetMobilityInsertionPerformance() to determine insertion benefit and cost, as well as the function CompareMobilityStrategies() to select better joining nodes. The function implementations for minimizing total energy consumption and maximizing system lifetime are shown in Figures 11 and 12, respectively. 


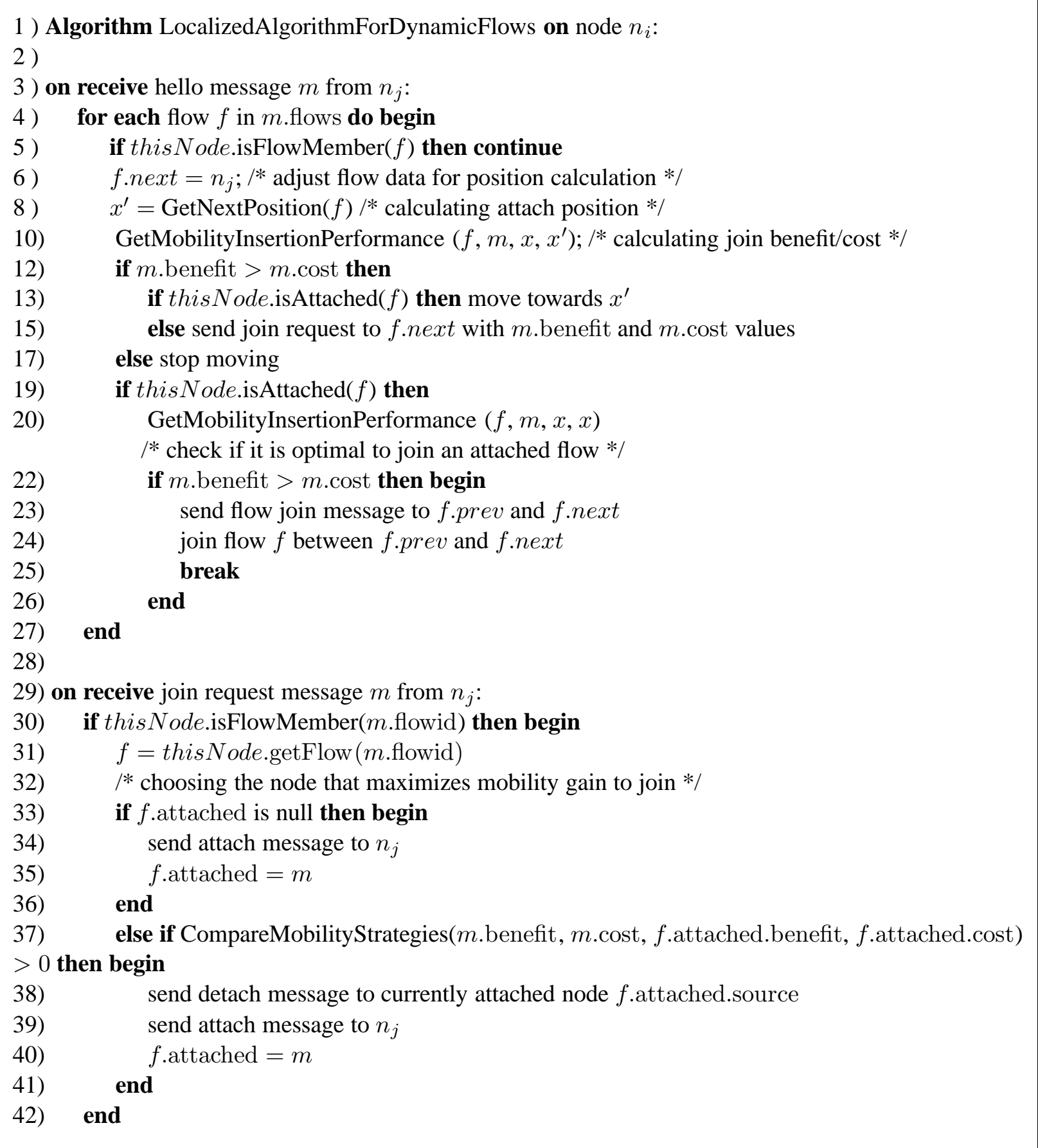

Figure 10. The localized algorithm for dynamic flows.

\section{The iMobif Framework}

We are now ready to describe iMobif, a practical framework for energy optimization under informed mobility. iMobif collects necessary node positions and residual energy information, and 
1 ) procedure GetMobilityInsertionPerformance (Flow $f$, inout Message $m$, Position $x$, Position $x^{\prime}$ )

2 ) begin

$3) \quad m$. benefit $=E_{T}\left(\mid f \cdot\right.$ prev.x - f.next.x $\left.\mid, \lambda_{f}\right)-E_{T}\left(\mid f \cdot\right.$ prev $\left.\cdot x-x^{\prime} \mid, \lambda_{f}\right)-E_{T}\left(\left|x^{\prime}-f \cdot n e x t . x\right|, \lambda_{f}\right)$

4 ) $\left.\quad m \cdot \operatorname{cost}=E_{M}\left(\left|x-x^{\prime}\right|\right)\right)$

5 ) end

6 )

7 ) function CompareMobilityStrategies

(Number benefit0, Number cost 0 , Number benefit 1 , Number $\cos t 1)$ : Integer

8 ) begin

9) return (benefit $0-\operatorname{cost} 0)-($ benefit $1-\operatorname{cost} 1)$

10) end

Figure 11. Minimizing total energy consumption using the generic algorithm for dynamic flows.

1 ) procedure GetMobilityInsertionPerformance (Flow $f$, inout Message $m$, Position $x$, Position $x^{\prime}$ )

2 ) begin

3) $\quad m$.benefit $=\min \left(\frac{e-E_{M}\left(\left|x-x^{\prime}\right|\right)}{E_{T}\left(\left|x^{\prime}-f \cdot n e x t . x\right|, 1\right)}, \frac{f \cdot p r e v \cdot e}{E_{T}\left(\mid f \cdot \text { prev. } x-x^{\prime} \mid, 1\right)}\right)$

4 ) $\quad m . \operatorname{cost}=\frac{\text { f.prev.e }}{E_{T}(\mid f \cdot \text { prev.x-f.next.x } \mid, 1)}$

5 ) end

6 )

7 ) function CompareMobilityStrategies

(Number benefit0, Number cost 0 , Number benefit 1 , Number cost 1 ) : Integer

8 ) begin

9) return $\max ($ benefit $0, \operatorname{cost} 0)-\max ($ benefit $1, \operatorname{cost} 1)$

10) end

Figure 12. Maximizing system lifetime using the generic algorithm for dynamic flows.

passes the information to the algorithms described in Section 4, which determine the proper mobility strategy.

\subsection{Overview}

In designing the iMobif framework, we make the following assumptions: 1) nodes are energy constrained; 2) nodes are mobile; 3) node movement consumes node energy; 4) nodes can select a transmission power level close to a specified value and use this power level for transmission; 5) nodes can measure residual node energy; 6) nodes can detect their locations; 7) nodes can move to location specified by software applications and protocols; 8) nodes can measure (or estimate from historical data) the energy needed to move to a target location; and 9) nodes can determine 
the minimum transmission power needed to communicate with nodes within a specific distance. Assumptions 1,2,3,4 specify the target environment of iMobif: mobile sensor or mobile ad hoc networks where nodes are battery-driven and transmission power is tunable. Assumption 5 holds for most nodes in such environment. Assumptions 6 and 7 require each node to be equipped with GPS or other positioning devices/algorithms [17]. Assumptions 8 and 9 are usually not supported by hardware. iMobif establishes the energy-distance relationship to provide required information.

Each iMobif node maintains the following information: 1) a list of mobility strategies and their corresponding functions; 2) the current location and residual energy of the node; 3) a neighbor table with the identity, location, and residual energy of each neighbor; 4) a routing table; 5) a flow table containing, for each flow traversing the node, its source, number of total data bits, previous node, mobility strategy and status, destination, and next node. According to Assumptions 5 and 6 above, a node can measure its current location and residual energy. Such information is placed in the corresponding tables. The routing table is managed by lower level routing protocols. In protocols such as AODV [18], for instance, each node periodically sends "hello" messages to probe and collect information from neighbors. In iMobif, a node in the system embeds in these "hello" messages its location, residual energy, and local flow table information, so that each node can obtain such information regarding its neighbors.

In the iMobif framework, the source of each flow determines current mobility strategy and status (enabled or disabled). The source selects a strategy from a mobility strategy list and enables or disables it according to feedback from the destination. The initial mobility status is both applicationand environment-specific. The iMobif framework is general in that it can be tuned to different energy optimization goals by changing the mobility strategy and the corresponding functions for node positioning, cost-benefit calculation, and information aggregation.

\subsection{Operations}

As described earlier, the flow source disseminates mobility strategy and status to other nodes along the flow path using message header of data packets. In addition, a source disseminates another flow parameter, the expected residual flow length, measured in data bits. The flow length information is used to calculate the benefit of the mobility, since a change in node location affects energy consumption of all subsequent packets. The flow length estimate is provided by the application. 
As described above, node mobility in iMobif framework is triggered by data packets in a packetby-packet fashion. The evaluation of overall performance is also packet-by-packet. This scheme ensures that the mobility is naturally synchronized without additional synchronization overhead. On the other hand, it implies that there may be oscillations if the cost-benefit combination produces disparate results between successive steps, resulting in a large number of notification packets. Although not formally proved, our simulation results show that only a few notification packets are sent for most flows, implying that the cost-benefit combination is consistent between successive steps.

In addition to processing data packets and sending "hello" messages, iMobif also sends back-toback probing packets to neighbors to determine energy-distance relationship. The probing packets are sent with decreasing transmission power, with the value of transmission power embedded in the packet. A neighbor overhearing these packets replies with an acknowledgement packet indicating the lowest transmission power of the probing packets it has received. Based on such feedback from different neighbors, a node can establish an energy-distance lookup table and derive the energydistance relationship and the parameter $\alpha$. This operation can be conducted with low frequency since channel characteristics are usually static.

\section{Performance Evaluation}

In this section we present evaluation results of the iMobif framework. First we describe the simulation setup, followed by simulation results for total energy consumption minimization and system lifetime maximization, respectively. Results for situations with and without topology constraints are presented, and the performance of global and localized algorithms are compared. Lastly we describe results that shed light on the internal processing of the framework, and evaluate the framework under practical energy constraints. We consider only a single one-to-one flow in this section, and leave the evaluation of multiple flows as future work.

\subsection{Simulation Setup}

We randomly distribute 100 nodes in a $100 \mathrm{~m} \times 100 \mathrm{~m}$ area. The communication range of each node is set to $20 \mathrm{~m}$. The resultant average number of neighbors per node is approximately 12 . We randomly select two nodes as the source and destination of the flow. The network uses greedy 
routing to forward packets from the source to the destination. We randomly set the initial residual energy of each node and the flow length; details are described in subsequent subsections. As described in Section 2.1, we use the transmission power model $P_{T}(d)=a+b d^{\alpha}$. We adopt parameter values similar to those in [13]. Specifically, we set $a=10^{-7} \mathrm{~J} / \mathrm{bit}, b=10^{-10} \mathrm{Jm}^{-\alpha} / \mathrm{bit}$, and vary the value of $\alpha$. We use the mobility cost model $E_{M}(d, l)=k d$, and vary the value of $k$. The maximum distance traveled is $1 \mathrm{~m}$ in each step. The flow rate is set to $1 \mathrm{KBps}(8 \mathrm{Kbps})$. In the simulations using iMobif, node mobility is initially disabled.

\subsection{Minimizing Total Energy Consumption}

Figures 13(a) and 13(b) show the location of the nodes in a typical flow before transmission and after the mobility strategy that is used to minimize total energy consumption reaches a steady state. The size of the circle representing each node is proportional to its residual energy. The figures show the iMobif framework is capable of reaching the optimal configuration using the localized approach. For this mobility strategy, the node location is independent of its residual energy.

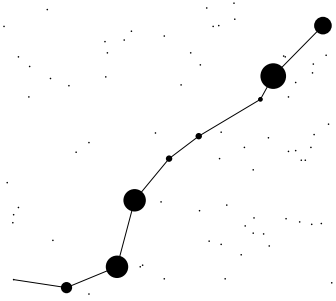

(a) original

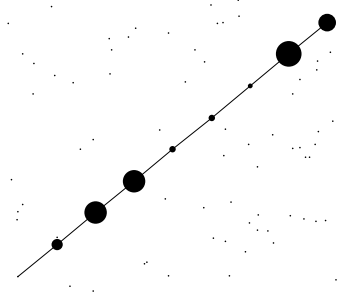

(b) the effect of the strategy that minimizes total energy consumption

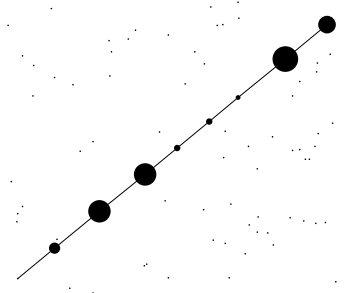

(c) the effect of the strategy that maximizes system lifetime

Figure 13. The effect of controlled mobility on a wireless network. The size of a node is proportional to its residual energy.

In Figure 14, we compare the total energy consumption of six approaches: an approach without node mobility, an approach with only cost-unaware mobility, the two approaches using the iMobif framework, which are both benefit- and cost-aware, and the two approaches using global algorithms. The static approaches do not change the composition of the flow, while the dynamic approaches may add new nodes to a flow path. We use the approach without mobility as the baseline, and define energy consumption ratio for the other five approaches as the ratio of the total 


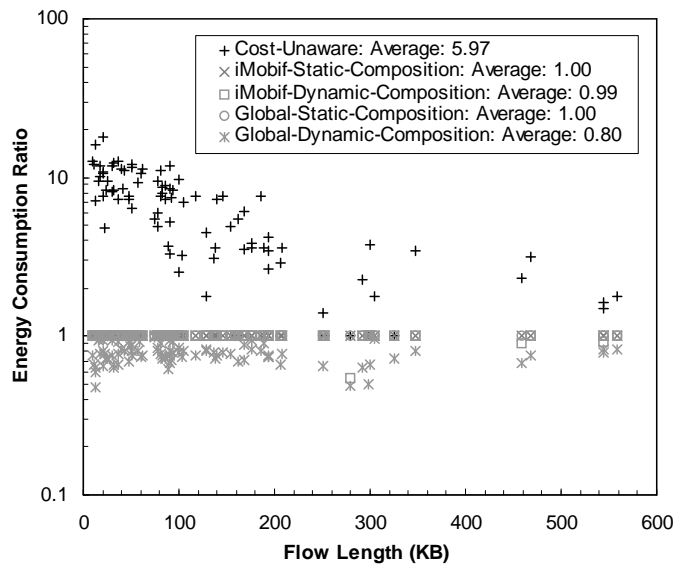

(a) $k=0.5, \alpha=3$, mean fbw length $100 \mathrm{~KB}$

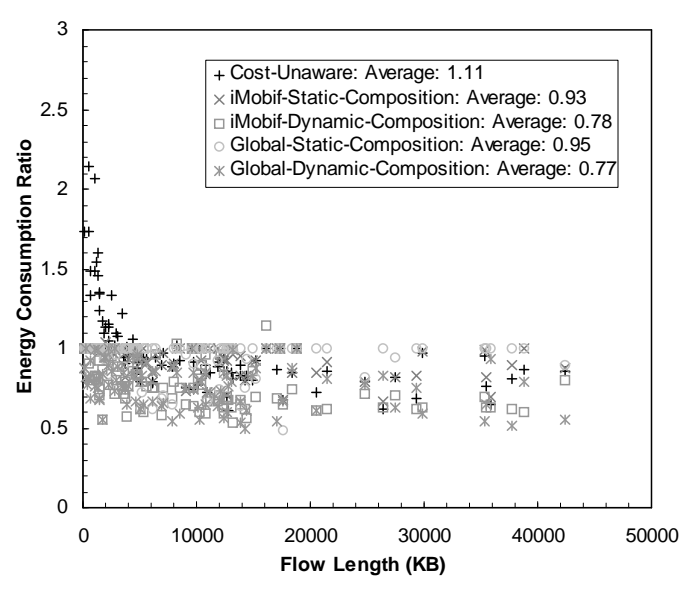

(c) $k=0.5, \alpha=3$, mean fbw length $10 \mathrm{MB}$

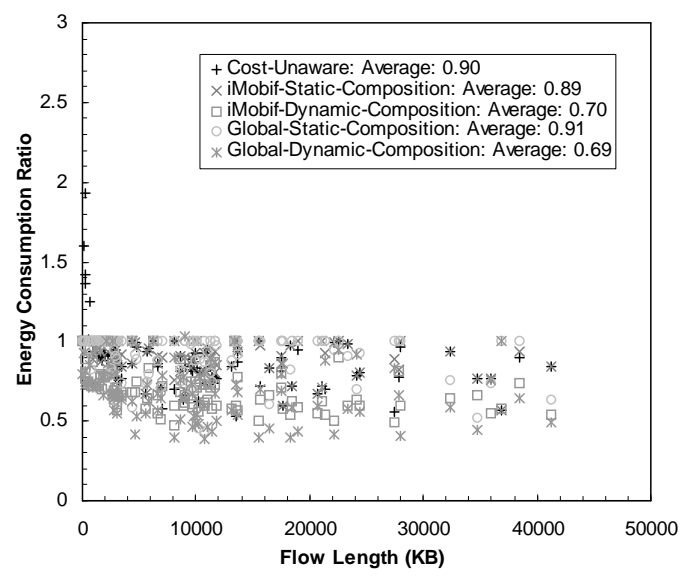

(e) $k=0.1, \alpha=3$, mean fbw length $10 \mathrm{MB}$

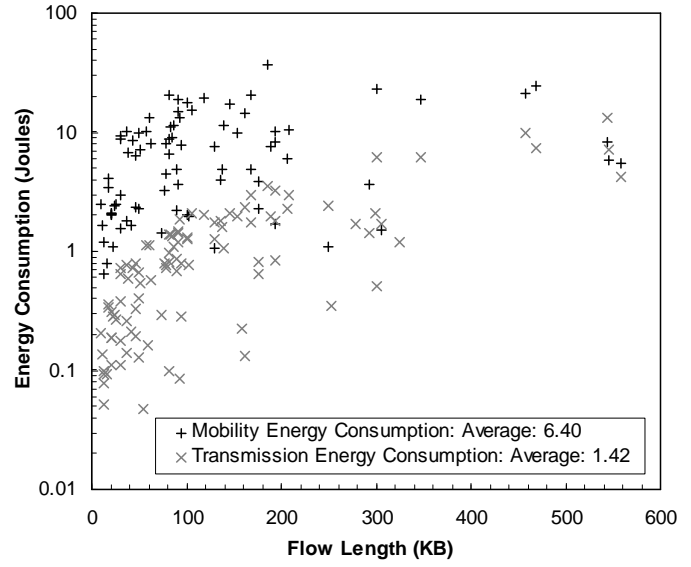

(b) comparing mobility and transmission cost

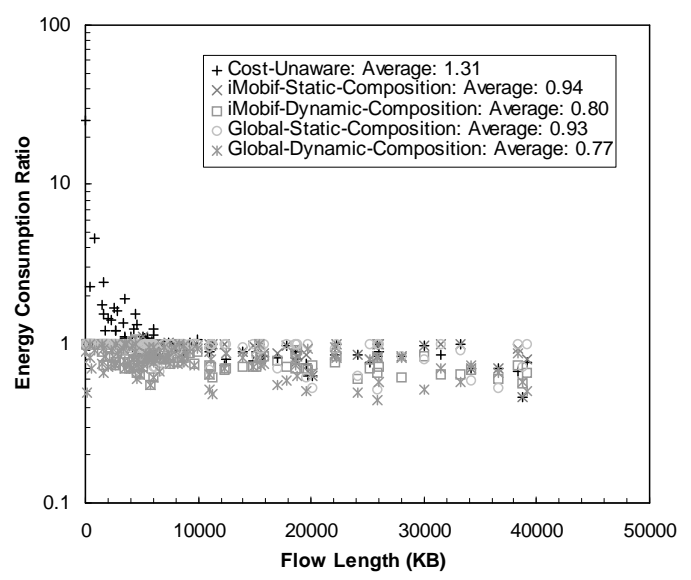

(d) $k=1, \alpha=3$, mean fbw length $10 \mathrm{MB}$

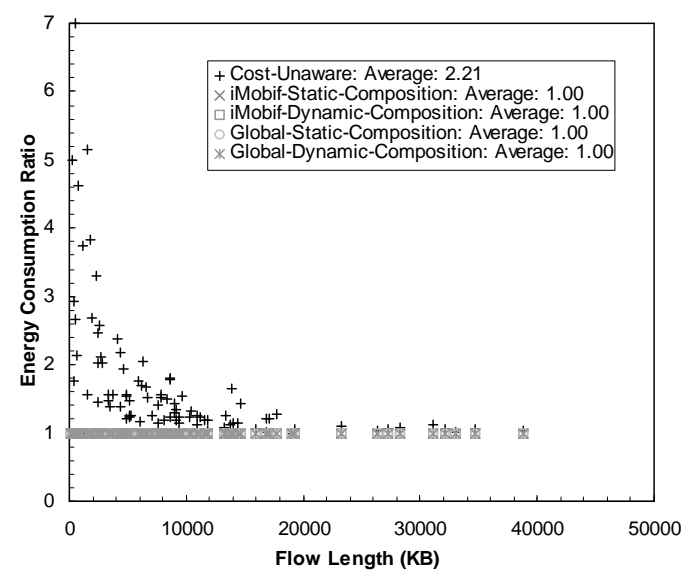

(f) $k=0.5, \alpha=2$, mean fbw length $10 \mathrm{MB}$

Figure 14. Performance comparison of the approaches for energy consumption reduction without topology constraint.

energy consumed to the energy consumed in the baseline approach. We expect the energy consumption of the five controlled-mobility approaches to be lower than that of the baseline approach. 
In other words, we expect the energy consumption ratio to be less than 1 . In addition, we expect the energy consumption ratio of the approaches using global algorithms to be less than that of iMobif approaches.

In the following tests, the flow length is exponentially distributed with mean $100 \mathrm{~KB}$ and $10 \mathrm{MB}$ respectively. The mobility cost parameter $k$ is set to $0.1,0.5$, and $1 \mathrm{~J} / \mathrm{m}$ respectively. Considering sensor nodes are usually small and light, such energy consumption level for node mobility is feasible. The transmission power parameter $\alpha$ is set to 2 and 3 respectively. We randomly generate 100 flows and collect the statistics.

First we present the results for mobility without topology constraints. Figure 14(a) shows that the energy consumption of the cost-unaware mobility approach is much higher than the baseline approach for short flows. As shown in Figure 14(b), this is due to the fact that the mobility cost is much higher than the transmission cost for short flows, and thus the mobility benefit is not enough to cover the cost. For longer flows, the cost-unaware mobility approach can reduce energy consumption, as shown in Figure 14(e). However, in most cases, the total energy consumption of the approach with cost-unaware mobility is higher than the approach without mobility. This result shows it is important to take mobility cost into consideration for practical applications. On the other hand, Figure 14 shows the approach using iMobif framework can achieve lower energy consumption than the approach without mobility for almost all flow instances. Even for flow instances that are long enough to cover the mobility cost, the performance of the iMobif approach is still comparable to that of the cost-unaware approach. This result indicates that the adverse impact of incorrect initial mobility status is limited. Figure 14 also shows the dynamic-composition approaches that insert new nodes into flows can further reduce energy consumption significantly.

The figures show the performance of iMobif approaches is comparable to that of the approaches using global algorithms in most cases except in Figure 14(a), where the global dynamiccomposition approach exhibits much better performance than its iMobif counterpart since the former has inserted much more new nodes into the flow. The latter has missed these candidates since node movement traces specified by the algorithm are suboptimal in this case. As shown, a global approach has the advantage of reducing node movement distance as well as locating more beneficial nodes for insertions, as long as its computed positions are really "optimal". The global algorithms described in Section 3 assume monotonic decrease of total energy consumption as nodes move to their "optimal" positions. Therefore, they only compare node energy consumption at the initial positions and the final positions, and make mobility decision based on the comparison re- 
sults. Our simulation results show, however, the energy decreasing is not necessarily monotonic. An "optimal" global algorithm may need to explore much more combinations of node positions to find the solution, otherwise it may produce no better results than the localized approaches. Moreover, a global approach requires the information on the number of nodes in the flow, which may be dynamic. In addition, the approach assumes all intermediate nodes can move to their optimal positions, which may not be the case due to a topology constraint or lack of mobility at some nodes. Instead, a localized algorithm does not require information on the number of in-flow nodes, and can "elegantly" handle all the cases where in-flow nodes are fixed or flow composition has been changed. Therefore, we claim iMobif is an appropriate solution to the informed mobility problems.

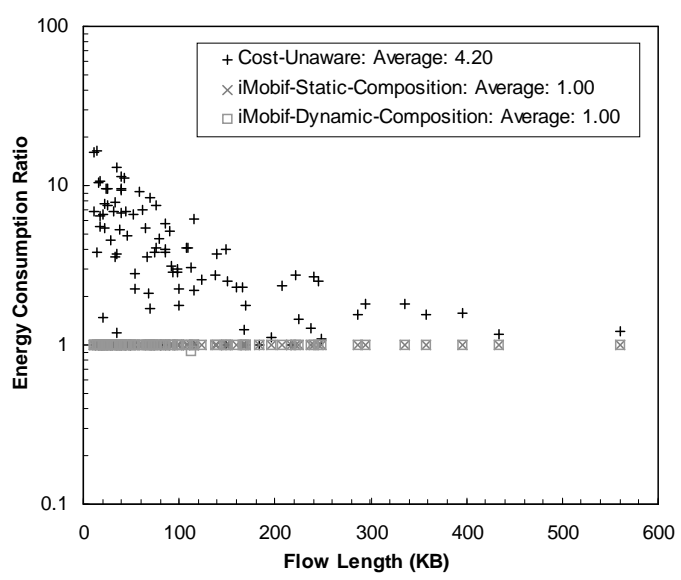

(a) $k=0.5, \alpha=3$, mean fbw length $100 \mathrm{~KB}$

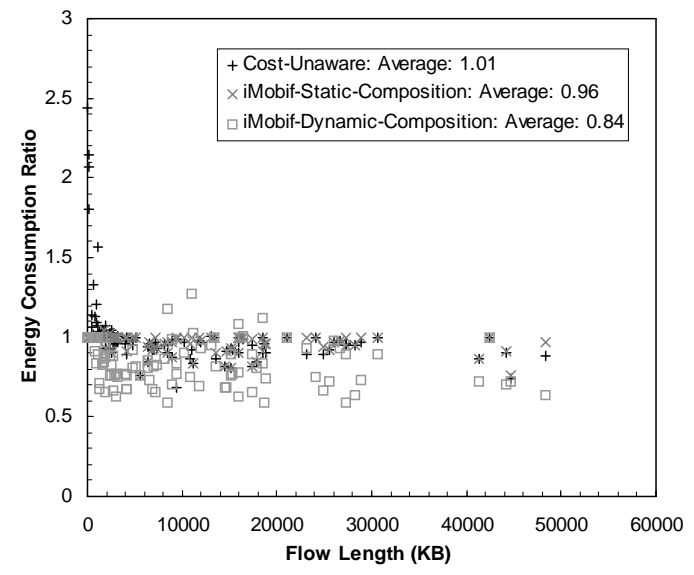

(b) $k=0.5, \alpha=3$, mean fbw length $10 \mathrm{MB}$

Figure 15. Performance comparison of the approaches for energy consumption reduction with topology constraint.

Next we present the results for mobility with the constraint that a node should not move outside of the transmission range of any of its current neighbors. Figure 15 shows, under this mobility constraint, the controlled mobility approaches are slightly less effective for energy consumption reduction than in the case of no mobility constraint. Therefore, the space for improvement over the cost-unware approach is smaller. However, iMobif framework still achieves lower energy consumption in most cases. 


\subsection{Maximizing System Lifetime}

Figure 13(c) shows the steady-state location of the nodes for the mobility strategy that maximizes system lifetime. Note that Figure 13(c) is actually different from Figure 13(b) although they appear similar. Clearly the distance between a node and the next node in the path is dependent on the node residual energy, indicating that the iMobif framework is effective in placing nodes at their optimal positions using the distributed algorithm.

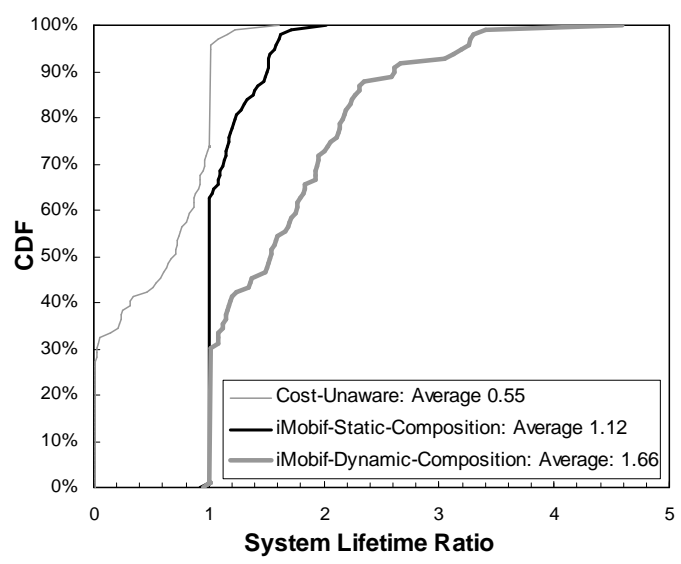

(a) without topology constraint

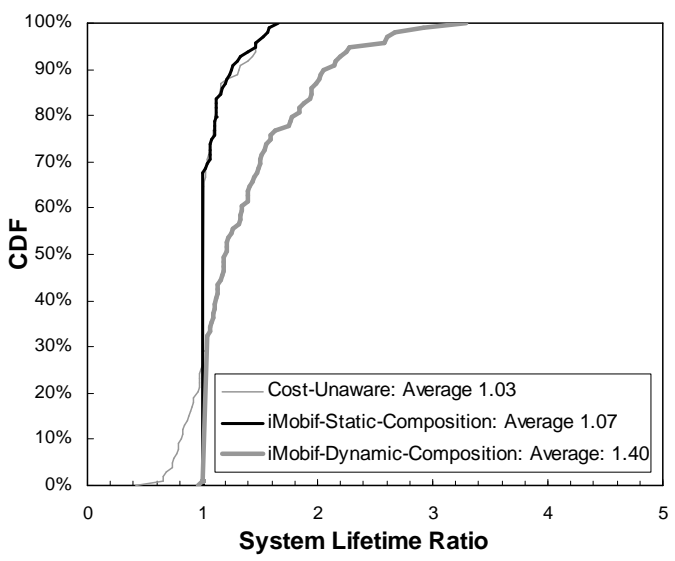

(b) with topology constraint

Figure 16. Performance comparison of the approaches for system lifetime increasing.

In Figure 16, we compare the system lifetime of the three approaches with and without the topology constraint. Again we select the approach without mobility as the baseline approach, and define system lifetime ratio for the other three mobility approaches as the ratio of the system lifetime to that of the baseline approach. The flow length is exponentially distributed with mean $10 \mathrm{MB}$, and the mobility cost parameter $k$ is set to 0.5 . The transmission power parameter $\alpha$ is set to 3 . The node residual energy is randomly chosen between 5 and 10 Joules (we intentionally set low residual energy to produce instances with short system lifetime). As Figure 16 shows, the system lifetime of the approach with cost-unaware mobility is usually shorter than the approach without mobility, since it is likely that the bottleneck nodes spend too much energy in moving to new locations. On the other hand, Figure 16 shows the approaches using the iMobif framework can achieve longer system lifetime than the approach without mobility for most flow instances. Although the average improvement is moderate, iMobif can increase system lifetime up to a factor of 2 for some flow instances with static flow composition, and up to a factor of 4 with dynamic flow 
composition. Even under the topology constraint, iMobif can extend system lifetime by factors of 1.6 and 3 for static and dynamic flow compositions, respectively. These results imply that the approximation algorithm is effective in reducing energy consumption at bottleneck nodes, thereby increasing system lifetime.

\subsection{Notifications and Insertions}

As described, iMobif uses a notification message sent from destination to source to change the status of the current mobility strategy. As shown in Figure 17(a), in the simulation traces the number of notification messages is small, indicating the cost-benefit comparison results are fairly consistent, and there are few oscillations. Again the flow length in this test is exponentially distributed with mean $10 \mathrm{MB}$. The mobility cost parameter $k$ is set to 0.5 , and the transmission power parameter $\alpha$ is set to 3 .

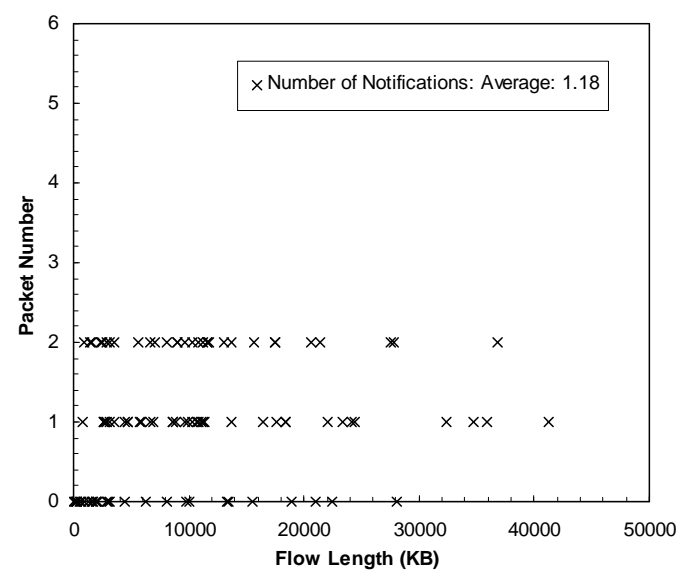

(a) the number of notifi cation messages

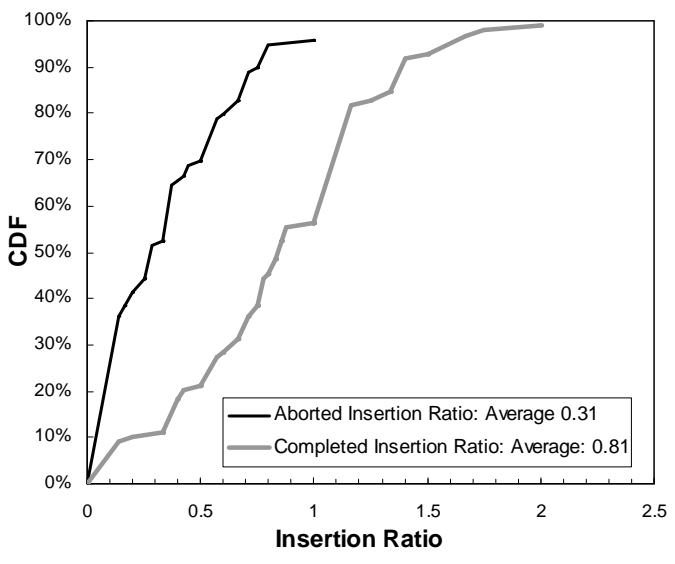

(b) the number of inserted nodes

Figure 17. The number of notifications and insertions.

The results in Sections 6.2 and 6.3 showed that adding new nodes to a flow path can significantly reduce energy consumption, or increase system lifetime, as desired. Next we study how many insertions are needed to achieve such performance gains. We define insertion ratio as the ratio of the number of nodes added relative to the original flow hop count. Figure 17(b) plots the CDF of the insertion ratio for 100 simulation runs without the topology constraint, with the parameters $k=0.1, \alpha=3$, and mean flow length 10MB. The insertion ratios for both aborted and completed insertions are shown. An insertion is aborted when a node is preliminarily attached to a flow, but 
never finishes joining the flow because the mobility cost becomes higher than the expected benefit. The figure shows many insertions are aborted, preventing further performance loss but causing the previous movement to be wasted. However, the number of completed insertions is much higher, so the overall benefit is still high enough to counterbalance the mobility cost.

\subsection{Impact of Practical Issues}

In real systems the transmission power level may not be continuously tunable. It is more common that a node provides several levels of transmission power and applications select one of them for transmission. To study the impact of transmission power granularity on the performance of iMobif, we conduct simulation with a granularity value of $1 \mathrm{dBm}$. The iMobif algorithms compute the transmission power needed based on the propagation distance. The power value is rounded up to the next discrete level, which is used for transmission. Figure 18(a) shows iMobif can achieve comparable energy consumption ratio under this realistic setting as the settings with continuously tunable transmission power. This result implies the energy consumption gap between different mobility strategies is usually substantial.

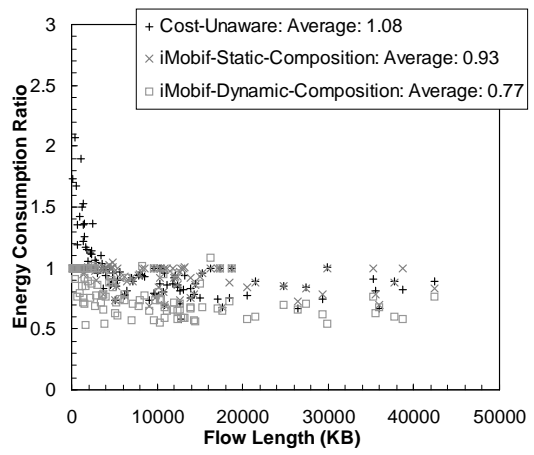

(a) $k=0.5, \alpha=3$, mean fbw length $10 \mathrm{MB}$, discrete transmission power

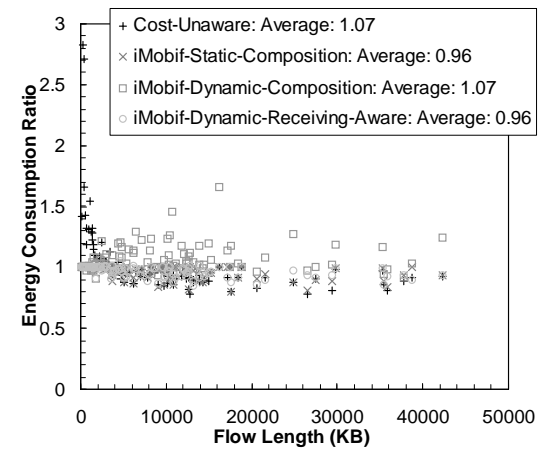

(b) $k=0.5, \alpha=3$, mean fbw length $10 \mathrm{MB}$, energy consumption under nontrivial receiving energy

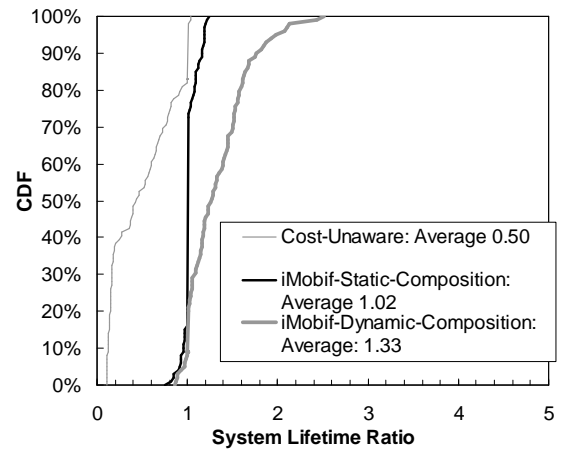

(c) $k=0.5, \alpha=3$, mean fbw length 10MB, system lifetime under non-trivial receiving energy

Figure 18. Performance comparison of the approaches under practical considerations.

iMobif can reduce transmission energy consumption by adjusting transmission power level. In reality, nodes also consume a fixed amount of energy for receiving messages. To study total energy consumption ratio of iMobif, we set the node receiving power fixed at $10 \mathrm{~mW}$ so that the energy consumption for receiving is approximately the same as the energy consumption for transmission 
in the baseline approach. Figure 18(b) shows the iMobif approach with fixed flow composition can still reduce total energy consumption, although with lower improvement. The approach with dynamic flow composition, however, may consume more energy than the baseline approach. This is due to the fact that inserted nodes introduce extra energy consumption for receiving. To solve this problem, we modify the function GetMobilityInsertionPerformance() in Figure 11 to include the expected receiving energy consumption into the cost of node insertion. The modified approach, labeled as "iMobif-Dynamic-Receiving-Aware" in Figure 18(b), exhibits higher performance than the baseline approach. On the other hand, Figure 18(c) shows iMobif approaches remain effective in maximizing system lifetime even if receiving energy consumption is considered. Extending the earlier analysis to include receiving energy consumption is a topic for future research.

\section{Related Work}

Controlled mobility has been exploited to increase sensor surveillance coverage $[11,12]$, recover disconnected networks [10], and reduce energy consumption [13] in wireless ad hoc and sensor networks. In [10], the authors proposed an approach that uses controlled mobility to improve communication reliability, specifically, to reconnect a partitioned sensor network. In [11,12], the authors proposed localized algorithms that increase sensor surveillance coverage by dispatching mobile sensor nodes to new locations not covered by existing sensor nodes. The localized nodeinsertion algorithm proposed in this paper is similar to the "auction"-based algorithms proposed in [12]. In addition to the above research, other research activities on controlled mobility in sensor networks are reported in [19]. However, most of these approaches do account for mobility cost. In this paper we consider energy consumption for both communication and mobility.

In [13], the authors proposed several approaches that adjust the network topology to reduce energy consumption. The application scenarios include single flow, multiple flows, and concast. The approaches are based on the observation that total energy consumption is minimized when all relaying nodes are evenly spaced on a straight line between the source and the destination. In these approaches, each relaying node moves to its ideal position to form the straight line with evenly spaced relays. The cost benefit tradeoff is addressed and it is shown that the energy consumption of signal propagation is quadratic (or higher) in the propagation distance, so the energy savings due to changing the locations of relaying nodes can be super-linear in the distance. On the other hand, the mobility cost, or the energy consumption for node movement, is linear in the distance. There- 
fore, the benefit of node movement will eventually outweigh the mobility cost in term of energy consumption, provided the lifetime of the flow is long enough. The above work, however, does not incorporate the cost-benefit tradeoff in the design of mobility strategies. iMobif uses similar distributed algorithms to optimize energy consumption using controlled mobility. However, iMobif also includes online cost-benefit comparisons, which cause the mobility strategy to be enabled or disabled dynamically so as to optimize system performance. In addition, iMobif provides for the insertion of new nodes into an existing flow, in order to further improve energy efficiency.

\section{Conclusions and Future Work}

In this paper we studied the informed mobility optimization problem. We designed localized algorithms to make mobility decisions using both mobility benefit and mobility cost. Based on these algorithms, we proposed iMobif, a framework to coordinate node mobility so as to optimize energy consumption in wireless ad hoc networks. The framework dynamically calculates the cost and benefit of a mobility strategy in a distributed fashion. The results of the cost-benefit comparison are used to enable or disable the mobility strategy. We demonstrated how to integrate two different mobility strategies into the framework: minimizing total energy consumption and maximizing system lifetime. Simulation results show iMobif is effective in reducing total energy consumption or increase system lifetime, compared to both an approach without mobility and an approach using only cost-unaware mobility.

The performance of iMobif depends on the relative weight between mobility cost and communication energy consumption. iMobif would be most effective in environments where node mobility consumes little power while communication is heavyweight in terms of energy consumption. In a sensor network, one of the target environments of iMobif, node mobility may be lightweight since sensor nodes are usually small and light. Although a flow between two nodes in sensor networks is usually short, the flow resulting from aggregation (of multiple simultaneous flows between a given node pair) or concatenation (of multiple successive flows between this node pair over a long interval) may be large. iMobif can be extended to treat aggregated and/or concatenated flows as a single long flow, and estimate its length based on historical data.

iMobif adjusts node positions to optimize energy consumption. The resultant network topology depends on the composition of, and the spatial and temporal relationship among, flows. An interesting extension of this work is to study the characteristics of the resultant topology and its impact on higher level applications and lower level network protocols. On the other hand, iMobif adjusts 
node positions using a flow-by-flow approach. A strategy optimal to individual flows is not necessarily optimal to the flows as a whole. Designing globally optimal strategies is challenging. It would also be interesting to study the impact of topology constraint on the efficiency of iMobif. Finally, it would be interesting to study how to extend iMobif to satisfy the requirements of specific applications by using a combination of controlled mobility, transmission power adaptation, flow pattern inference, and mobility collaboration.

\section{References}

[1] S. Bandyopadhyay and E. J. Coyle, "An energy effi cient hierarchical clustering algorithm for wireless sensor networks," in Proceedings of IEEE INFOCOM, 2003.

[2] A. Cerpa and D. Estrin, "Adaptive self-confi guring sensor networks topologies," in Proceedings of INFOCOM, June 2002.

[3] Y.-C. Tseng, Y.-N. Chang, and P.-H. Tseng, 'Energy-effi cient topology control for wireless ad hoc sensor networks," in Proceedings of International Computer Symposium (ICS), 2002.

[4] J. Pan, Y. T. Hou, L. Cai, Y. Shi, and S. X. Shen, 'Topology control for wireless sensor networks," in Proceedings of the 9th annual international conference on Mobile computing and networking (MobiCom), September 2003.

[5] K. Dantu, M. Rahimi, H. Shah, S. Babel, A. Dhariwal, and G. Sukhatme, 'Robomote: Enabling mobility in sensor networks,” Tech. Rep. CRES-04-006, Department of Computer Science, University of Southern California, 2004.

[6] J. Wu and F. Dai, 'Mobility-sensitive topology control in mobile ad hoc networks," in Proceedings of IPDPS, 2004.

[7] S. Chakraborty, D. Yau, and J. Lui, 'On the effectiveness of movement prediction to reduce energy consumption in wireless communication (extended abstract),"in Proceedings of SIGMETRICS, June 2003.

[8] M. Grossglauser and D. Tse, 'Mobility increases the capacity of ad-hoc wireless networks," in Proceedings of INFOCOM, pp. 1360-1369, March 2000.

[9] S. Capkun, J.-P. Hubaux, and L. Buttyan, "Mobility helps security in ad hoc networks,"in Proceedings of the 4th ACM international symposium on Mobile ad hoc networking and computing (MobiHoc), June 2003.

[10] T. Srinidhi, G. Sridhar, and V. Sridhar, "Topology management in ad hoc mobile wireless networks," in Proceedings of Real-Time Systems Symposium, Work-in-Progress Session, December 2003.

[11] G. Wang, G. Cao, and T. L. Porta, 'Movement-assisted sensor deployment," in Proceedings of IEEE INFOCOM, March 2004.

[12] G. Wang, G. Cao, and T. L. Porta, "A bidding protocol for deploying mobile sensors," in Proceedings of ICNP, November 2003.

[13] D. Goldenberg, J. Lin, A. S. Morse, B. Rosen, and Y. R. Yang, "Towards mobility as a network control primitive," in Proceedings of MobiHoc, 2004.

[14] A. Chakrabarti, A. Sabharwal, and B. Aazhang, "Using Predictable Observer Mobility for Power Effi cient Design of Sensor Networks," in Information Processing in Sensor Networks, (Palo Alto, CA), April 2003.

[15] S. Jain, R. C. Shah, G. Borriello, W. Brunette, and S. Roy, 'Exploiting mobility for energy effi cient data collection in sensor networks," in Proceedings of Wiopt, March 2004.

[16] C. Tang and P. K. McKinley, 'MMobif: An informed mobility framework for energy optimization in wireless ad hoc networks," in Proceedings of the Second International Workshop on Wireless Ad Hoc Networking (WWAN 2005, to appear), June 2005.

[17] L. Hu and D. Evans, 'Localization for mobile sensor networks," in Proceedings of MobiCom, September 2004.

[18] C. E. Perkins and E. M. Royer, "Ad hoc on-demand distance vector routing," in Proceedings of the 2nd IEEE Workshop on Mobile Computing Systems and Applications, pp. 90-100, February 1999.

[19] C. Tang, 'Mobility-assisted sensing and communication in sensor networks." available at http://www.cse.msu.edu/tangchip/publication/sensor_mobility.pdf. 